\title{
Method of Generating Transient Equivalent Sink and Test Target Temperatures for Swift BAT
}

\author{
Michael K. Choi* \\ NASA Goddard Space Flight Center, Greenbelt, MD 20771
}

The NASA Swift mission has a $600-\mathrm{km}$ altitude and a $22^{\circ}$ maximum inclination. The sun angle varies from $45^{\circ}$ to $180^{\circ}$ in normal operation. As a result, environmental heat fluxes absorbed by the Burst Alert Telescope (BAT) radiator and loop heat pipe (LHP) compensation chambers (CCs) vary transiently. Therefore the equivalent sink temperatures for the radiator and CCs varies transiently. In thermal performance verification testing in vacuum, the radiator and CCs radiated heat to sink targets. This paper presents an analytical technique for generating orbit transient equivalent sink temperatures and a technique for generating transient sink target temperatures for the radiator and LHP CCs. Using these techniques, transient target temperatures for the radiator and LHP CCs were generated for three thermal environmental cases: worst hot case, worst cold case, and cooldown and warmup between worst hot case in sunlight and worst cold case in the eclipse, and three different heat transport values: $128 \mathrm{~W}, 255 \mathrm{~W}$, and $382 \mathrm{~W}$. The $128 \mathrm{~W}$ case assumed that the two LHPs transport $255 \mathrm{~W}$ equally to the radiator. The $255 \mathrm{~W}$ case assumed that one LHP fails so that the remaining LHP transports all the waste heat from the detector array to the radiator. The $382 \mathrm{~W}$ case assumed that one LHP fails so that the remaining LHP transports all the waste heat from the detector array to the radiator, and has a 50\% design margin. All these transient target temperatures were successfully implemented in the engineering test unit (ETU) LHP and flight LHP thermal performance verification tests in vacuum.

\section{Nomenclature}

$\begin{array}{ll}\text { BAT } & =\text { Burst Alert Telescope } \\ \mathrm{CC} & =\text { compensation chamber of loop heat pipe } \\ \mathrm{CCHP} & =\text { constant conductance heat pipe } \\ G_{f, l h p} & =\text { radiation coupling between LHP CC and space and spacecraft in flight } \\ G_{f, r} & =\text { radiation coupling between radiator and space and spacecraft in flight } \\ G_{t, l h p} & =\text { radiation coupling between LHP CC and target in test } \\ G_{t, r} & =\text { radiation coupling between radiator and target in test } \\ \mathrm{LHP} & =\text { loop heat pipe } \\ \mathrm{MLI} & =\text { multi-layer insulation } \\ Q_{i n t} & =\text { LHP CC internal heat transfer } \\ Q_{r} & =\text { heat transported by LHP(s) to radiator } \\ \sigma & =\text { Stefann-Boltzmann constant } \\ T_{e, l h p} & =\text { LHP CC equivalent sink temperature } \\ T_{e, r} & =\text { radiator equivalent sink temperature } \\ T_{l h p} & =\text { LHP CC temperature } \\ T_{r} & =\text { equivalent radiator temperature } \\ T_{t, l h p} & =\text { LHP CC target temperature in test } \\ T_{t, r} & =\text { radiator target temperature in test }\end{array}$

\footnotetext{
" Swift Thermal Systems Lead Engineer, Thermal Engineering Branch/Code 545, AIAA Associate Fellow. 1 


\section{Introduction}

THE Burst Alert Telescope (BAT) is an instrument on the NASA Swift spacecraft. It is developed by Goddard Space Flight Center (GSFC). It is designed to detect gamma ray burst over a broad region of the sky and quickly align the telescopes on the spacecraft to the gamma ray source. The thermal requirements for the BAT detector arrays are very stringent. The maximum allowable temperature gradient of the 256 cadmium zinc telluride (CZT) detectors is $1^{\circ} \mathrm{C}$. Also, the maximum allowable rate of temperature change of the ASICs of the 256 Detector Modules (DMs) is $1^{\circ} \mathrm{C}$ on any time scale. ${ }^{1}$ The total power dissipation of the DMs, BVRs, XA1VRs and BCDHs is $208 \mathrm{~W}$ measured. Eight constant conductance heat pipes (CCHPs) embedded in the Detector Array Plate (DAP) minimize the temperature gradient of the DMs. Two LHPs transport the waste heat from the embedded CCHPs to the radiator (see Fig. 1) ${ }^{2}$ The flight radiator consists of thirty-six $4.064 \mathrm{~cm}$ (1.6 in) deep pockets, for mechanical reasons (see Fig. 2). AZW-LA-II white paint is the radiator thermal coating. The DMs are cold biased, and active heater control is used to meet the temperature gradient and stability requirements. The orbit average heater power prediction is $32 \mathrm{~W}$. Therefore the LHPs are required to transport a total of $240 \mathrm{~W}$ of waste heat to the radiator during the operational mode. In designing and manufacturing the LHPs, the transport capacity of each LHP was specified to be $382 \mathrm{~W}$ so that there is a $50 \%$ margin for a calculated heat dissipation of $255 \mathrm{~W}$ before the detector array was built. If the LHPs share the heat transport equally, each LHP transports $128 \mathrm{~W}$. If one LHP failed, the remaining unit will transport all $255 \mathrm{~W}$.

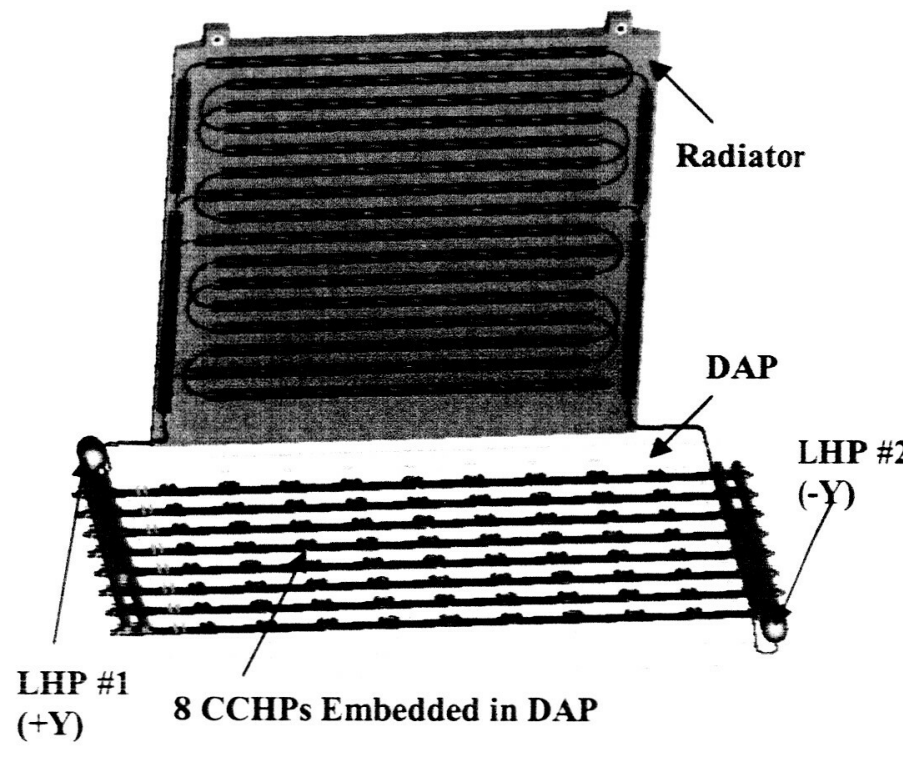

Figure 1. BAT LHP Thermal System Schematic.

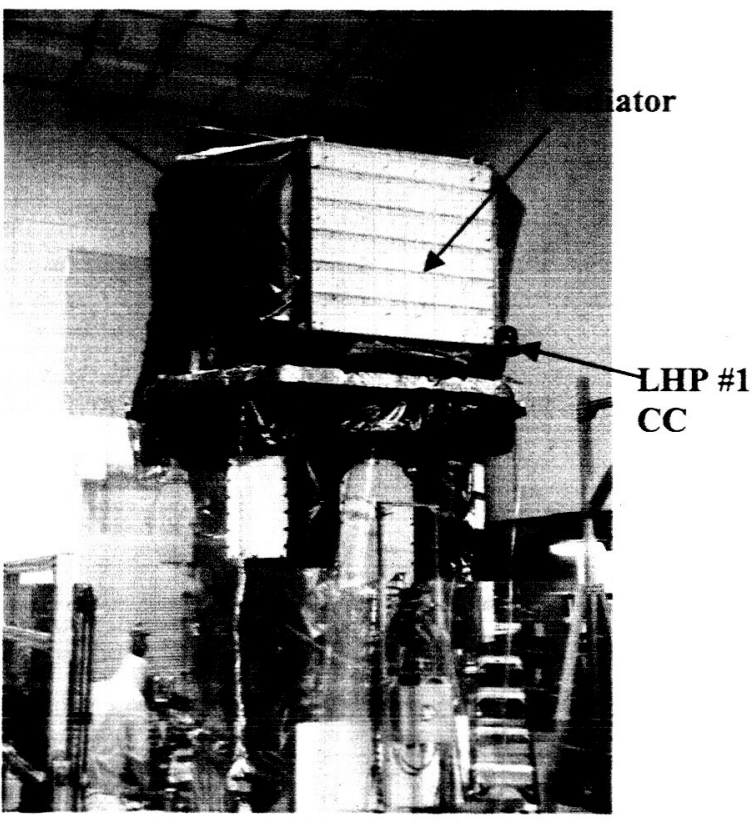

Figure 2. BAT on Observatory.

The Swift mission has a $600-\mathrm{km}$ altitude and a $22^{\circ}$ maximum inclination. The sun angle varies from $45^{\circ}$ to $180^{\circ}$ in the normal operation mode. The environmental heat fluxes absorbed by the BAT radiator vary transiently. Therefore, equivalent sink temperatures for the BAT radiator vary transiently. A thermal performance verification test in vacuum was performed on the LHP engineering test unit (ETU), before the flight units were built. In this test, a radiator ETU was used. It has A276 white paint as the thermal control coating. It radiated heat to a heat sink target, which has Z306 black paint as the thermal coating. After the flight LHPs were built, they were integrated to the flight radiator that has AZ-Tek's AZW-LA-II white paint as the thermal coating. A thermal performance verification test in vacuum was performed on the LHPs and radiator assembly before it was integrated to the BAT telescope assembly. The LHP CCs have 5-mil silver Teflon as the thermal control coating. The environmental heat fluxes absorbed by the CCs vary transiently. Therefore, equivalent sink temperatures for the CCs vary transiently. The temperatures of the CCs are actively controlled by heaters and electronics heater controllers with flight adjustable set points to meet the temperature stability requirement of the detector array.

Since the equivalent sink temperature of the BAT radiator change transiently, the radiator temperature also changes transiently. It was necessary to see how the LHP thermal performance is affected by the transient radiator temperature. Also it was necessary to see how the heater controller controllability and heater duty cycles are affected by the transient $\mathrm{CC}$ sink temperatures. An analytical technique was developed to generate transient equivalent sink 
temperatures for the BAT radiator and LHP CCs. Also an analytical technique was developed to generate transient target temperatures for the radiator and LHP CCs in thermal vacuum performance tests. This paper presents these techniques.

\section{Technique of Generating Equivalent Sink Temperature}

An instrument module and optical bench integrated thermal model was developed at GSFC. The instrument module consists of three instruments - BAT, X-Ray Telescope (XRT) and Ultraviolet Optical Telescope (UVOT). A simplified spacecraft bus thermal model was also included in the integrated model. Figure 3 shows the Geometric Mathematical Model (GMM) in Thermal Synthesizer System (TSS) format of the integrated thermal model. It was used to generate environmental heat fluxes and radiation couplings. An absorptance of 0.09 and an emittance of 0.91 at beginning of life were used for AZW-LA-II white paint. An absorptance of 0.13 and emittance of 0.88 at end of life were used. The sun angle and beta angle were varied parametrically to determine the worst hot case and worst cold case values for the BAT radiator. Figure 4 presents the effect of sun angles on the worst hot case absorbed flux for the radiator. It can be seen that the worst hot case sun angle is $90^{\circ}$ in sunlight and $180^{\circ}$ in the eclipse. Figure 5 presents the effect of sun angles on the worst cold case absorbed flux for the radiator. It can be seen that the worst cold case sun angle is $180^{\circ}$ in sunlight and $90^{\circ}$ in the eclipse. An absorptance of 0.08 and an emittance of 0.78 at beginning of life were used for 5-mil silver Teflon. An absorptance of 0.20 and emittance of 0.74 at end of life were used. The sun angle and beta angle were also varied parametrically to determine the worst hot case and worst cold case values for the LHP CCs.

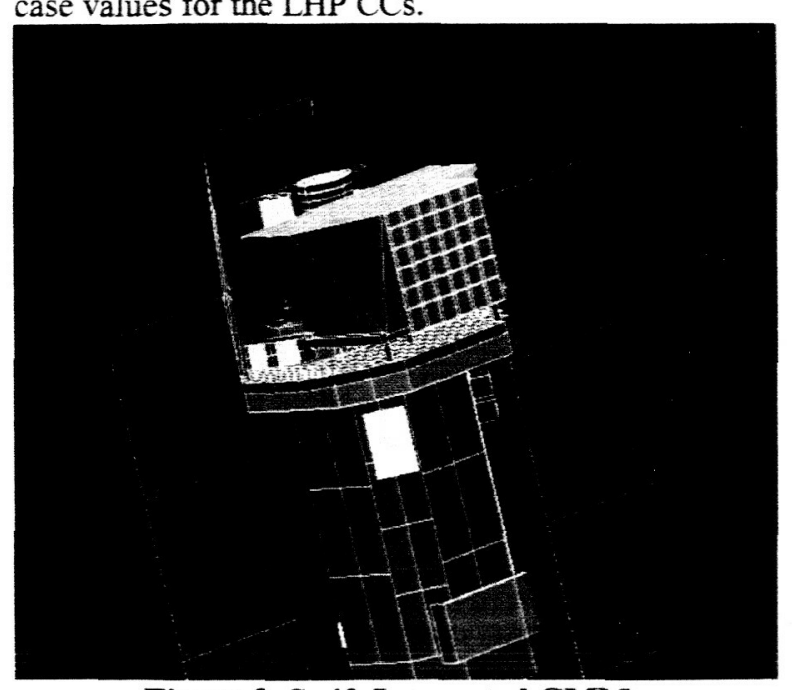

Figure 3. Swift Integrated GMM.

The worst hot case or worst cold case environmental heat fluxes absorbed and radiation couplings were then used in the Thermal Mathematical Model (TMM), which is in SINDA/FLUINT format. The analytical technique to generate equivalent sink temperatures for the BAT radiator is as follows:

-All the radiator nodes are arithmetic nodes.

-All the radiator nodes have no power dissipation.

- All the radiator nodes have no conduction couplings.

-No heat radiation through the radiator MLI blankets.

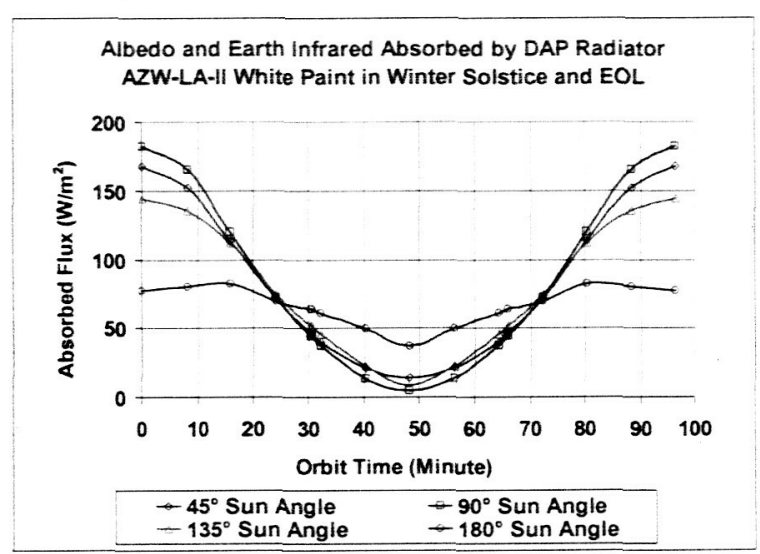

Figure 4. Worst Hot Case Absorbed Flux for BAT Radiator.

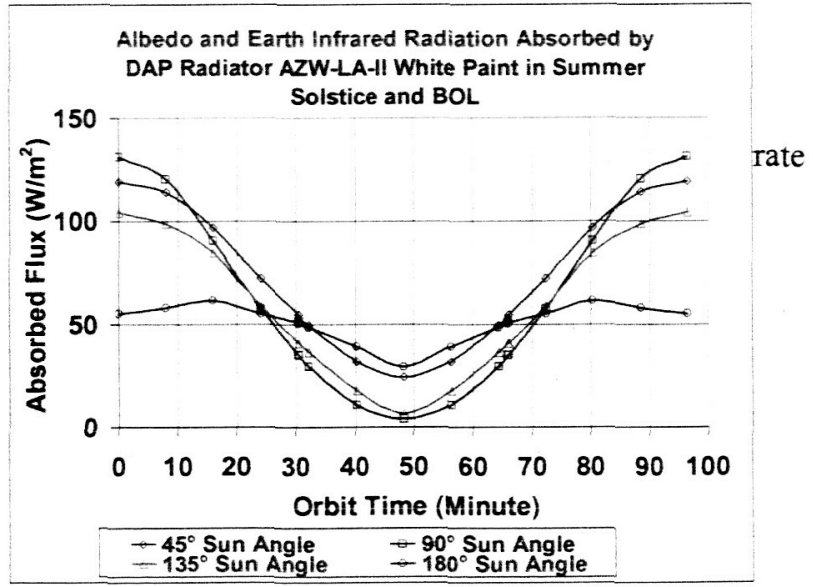

Figure 5. Worst Cold Case Absorbed Flux for BAT Radiator.

\section{Results of Generating Equivalent Sink Temperatures}

Equivalent sink temperatures were generated for three cases: 1) worst hot case, 2) worst cold case, and 3) cooldown and warmup between worst hot case in sunlight and worst cold case in the eclipse. Case \#3 was to 
simulate a sudden change in thermal environment from the worst hot sun angle in sunlight to the worst cold sun angle in the eclipse due to spacecraft slewing.

\section{A. Worst Hot Case}

Figure 6 presents the worst hot case transient equivalent sink temperatures for the BAT radiator and LHP CCs. It can be seen that LHP \#2 CC is warmer than LHP \#1 CC. This was expected because LHP \#2 CC is located near the rear $(+Z)$ end of the BAT and it has a smaller view factor to space, and has a significant view factor to the sunshade.

\section{B. Worst Cold Case}

Figure 7 presents the worst hot case transient equivalent sink temperatures for the BAT radiator and LHP CCs. It can be seen that LHP \#1 CC is colder than LHP \#2 CC because it has a larger view factor to space, and its view factor to the sunshade is negligible.

\section{Cooldown and Warmup between Worst Hot Case in Sunlight and Worst Cold Case in Eclipse}

Figure 8 presents the cooldown and warmup equivalent sink temperatures between the worst hot case in sunlight and worst cold case in eclipse for the BAT radiator and LHP CCs.

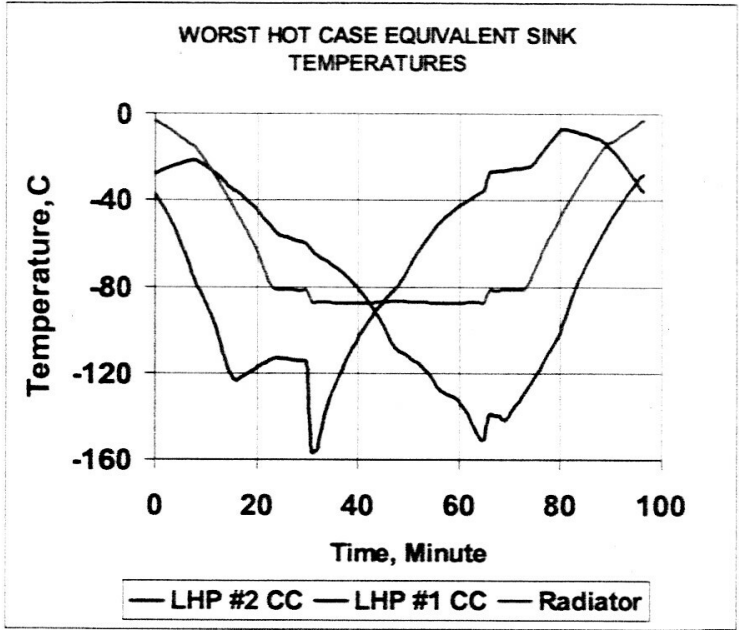

Figure 6. Worst Hot Case Equivalent Sink Temperatures.

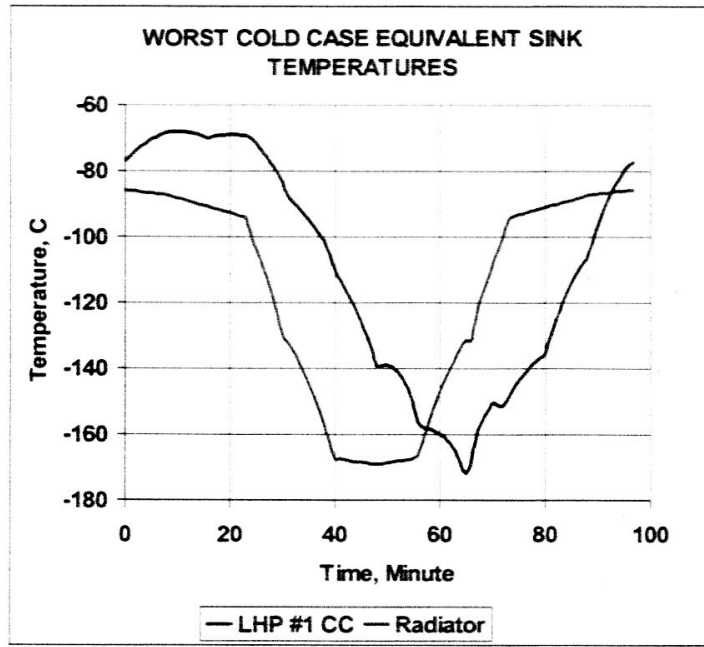

Figure 7. Worst Cold Case Equivalent Sink Temperatures.

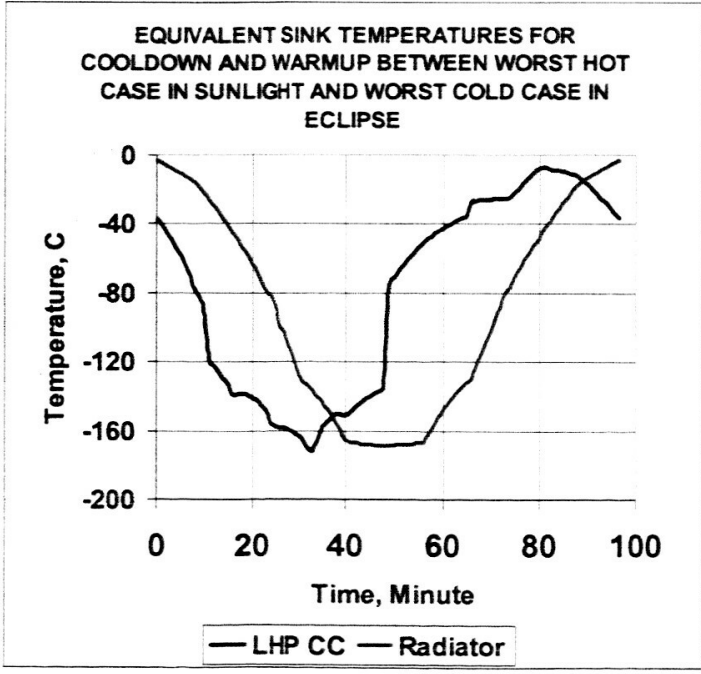

Figure 8. Equivalent Sink Temperatures for Cooldown and Warmup between Worst Hot Case in Sunlight and Worst Cold Case in Eclipse. 


\section{Technique of Generating Test Target Temperatures}

The anaiytical technique of generating the radiator and LHP CC target temperatures consists of two steps, which are described below. A target is a Ground Support Equipment, such as a cryopanel or thermal shroud, which simulates the heat sink of the radiator or LHP CCs in a thermal vacuum test.

\section{A. Step 1: Computation of Equivalent Radiator Temperatures}

Using the transient equivalent sink temperature, heat transported by the LHP(s) to the radiator, and the radiation coupling from the radiator to space and spacecraft, the transient equivalent radiator temperature is computed by using the following equation and a Microsoft EXCEL spreadsheet:

$$
Q_{r}=\sigma G_{f, r}\left(T_{r}^{4}-T_{e, r}{ }^{4}\right)
$$

where $Q_{r}=$ heat transported by $\operatorname{LHP}(\mathrm{s})$ to radiator,

$\sigma=$ Stefann-Boltzmann constant,

$G_{f, r}=$ radiation coupling between radiator and space and spacecraft,

$T_{r}=$ equivalent radiator temperature,

$T_{e, r}=$ radiator equivalent sink temperature.

Rearranging Eq. (1), we have:

$$
T_{r}=\left[Q_{r} /\left(\sigma G_{f, r}\right)+T_{e . r}{ }^{4}\right]^{0.25}
$$

Because the LHP CC temperatures are actively controlled by heaters and heater controllers, the LHP CC temperatures are maintained at the heater controller set point plus or minus a tolerance of $0.5^{\circ} \mathrm{C}$ or smaller. The equivalent radiator temperature is replaced by the LHP CC temperature. An energy balance on the LHP CC in flight is as follows:

$$
Q_{i n t}=\sigma G_{f . l h p}\left(T_{l h p}{ }^{4}-T_{e . l h p}{ }^{4}\right)
$$

where $Q_{\text {int }}=$ LHP CC internal heat transfer,

$\sigma=$ Stefann-Boltzmann constant,

$G_{f, l h p}=$ radiation coupling between LHP CC and space and spacecraft,

$T_{\text {lhp }}=$ LHP CC temperature,

$T_{e, l h p}=$ LHP CC equivalent sink temperature.

The temperature gradient between the BAT XA1 ASIC and LHP CC was predicted to be $9^{\circ} \mathrm{C}$. The maximum allowable flight temperature of the XA1 ASIC is $20^{\circ} \mathrm{C}$. Therefore the heater controller set point, $T_{i h n}$, for the LHP $\mathrm{CCs}$ is $11^{\circ} \mathrm{C}$ in the worst hot case. The minimum set point of the heater controller is $-15^{\circ} \mathrm{C}$. Therefore the heater controller set point, $T_{l h p}$, for the LHP CCs in the worst cold case is $-15^{\circ} \mathrm{C}$.

\section{B. Step 2: Computation of Target Temperatures}

Using the transient equivalent radiator temperature, heat transported by the LHP(s) to the radiator, and the radiation coupling between the radiator and target from TSS, the transient equivalent target temperature is computed by using the following equation and a Microsoft EXCEL spreadsheet:

$$
Q_{r}=\sigma G_{t, r}\left(T_{r}^{4}-T_{t, r}^{4}\right)
$$

where $Q_{r}=$ heat transported to radiator,

$\sigma=$ Stefann-Boltzmann constant,

$G_{t, r}=$ radiation coupling between radiator and target,

$T_{r}=$ equivalent radiator temperature computed from Step 1 ,

$T_{t, r}=$ radiator target temperature.

Rearranging Eq. (3), we have

$$
T_{t, r}=\left[T_{r}^{4}-Q_{r} /\left(\sigma G_{t, r}\right)\right]^{0.25}
$$


An energy balance on the LHP CC in the thermal vacuum performance verification test is as follows:

$$
Q_{i n t}=\sigma G_{t . h p}\left(T_{i h p}{ }^{4}-T_{t . i h p}{ }^{4}\right)
$$

where $Q_{\text {int }}=$ LHP CC internal heat transfer,

$\sigma=$ Stefann-Boltzmann constant,

$G_{t, l h p}=$ radiation coupling between LHP CC and target,

$T_{\text {Ihp }}=$ LHP CC temperature,

$T_{t, l h p}=$ LHP CC target temperature.

To maintain $Q_{i n t}$ in the test the same as that in flight, Eq. (3) and Eq. (6) are equated:

$$
\sigma G_{f, l h p}\left(T_{l h p}{ }^{4}-T_{e, l h p}{ }^{4}\right)=\sigma G_{t, l h p}\left(T_{l h p}{ }^{4}-T_{t, l h p}{ }^{4}\right)
$$

Simplifying Eq. (7), we have:

$$
T_{t . l h p}=\left\{\left(G_{f . l h p} / G_{t . l h p}\right) T_{e . l h p}{ }^{4}+\left[1-\left(G_{f . l h p} / G_{t, l h p}\right)\right] T_{l h p}{ }^{4}\right\}^{0.25}
$$

\section{Results of Generating Test Target Temperatures}

The results of generating the radiator target and LHP CC target temperatures are presented below.

\section{A. Worst Hot Case}

Figure 9 presents the worst hot case radiator target temperatures for the BAT radiator, comparing three values of heat transport by the LHP to the radiator. For the $128 \mathrm{~W}$ case, it was assumed that the two LHPs transport $255 \mathrm{~W}$ equally to the radiator. For the $255 \mathrm{~W}$ case, it was assumed that one LHP fails so that the remaining LHP transports all the waste heat from the embedded CCHPs to the radiator. For the $382 \mathrm{~W}$ case, it was assumed that one LHP fails so that the remaining LHP transports all the waste heat from the embedded CCHPs to the radiator, and has a $50 \%$ design margin. It can be seen that the heat transport by the LHP to the radiator has a significant effect on the radiator target temperature. As the heat transport increases, the radiator target temperature decreases.

Figure 10 presents the worst hot case target temperatures for the LHP CCs. The LHP CC heater controllers were set to $11^{\circ} \mathrm{C}$ in the worst hot case.

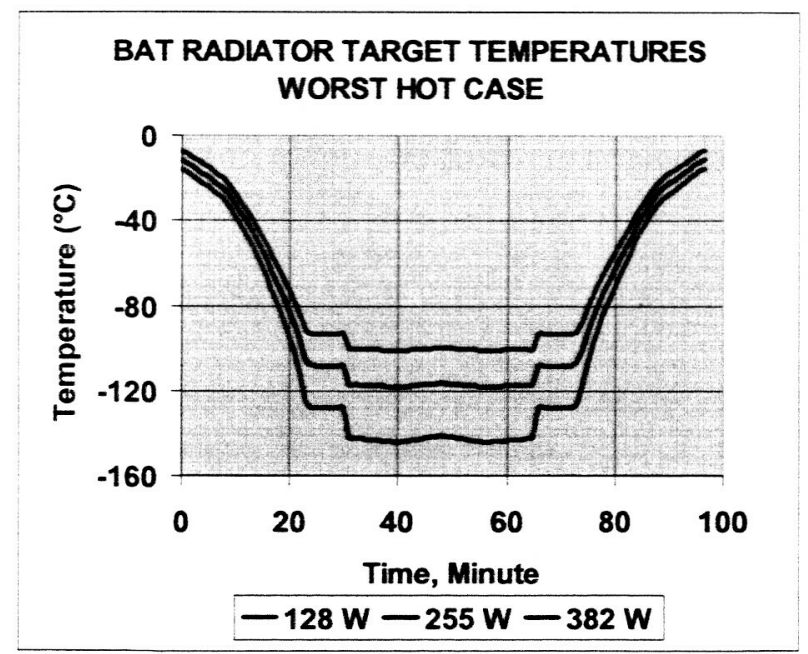

Figure 9. Worst Hot Case BAT Radiator Target Temperatures.

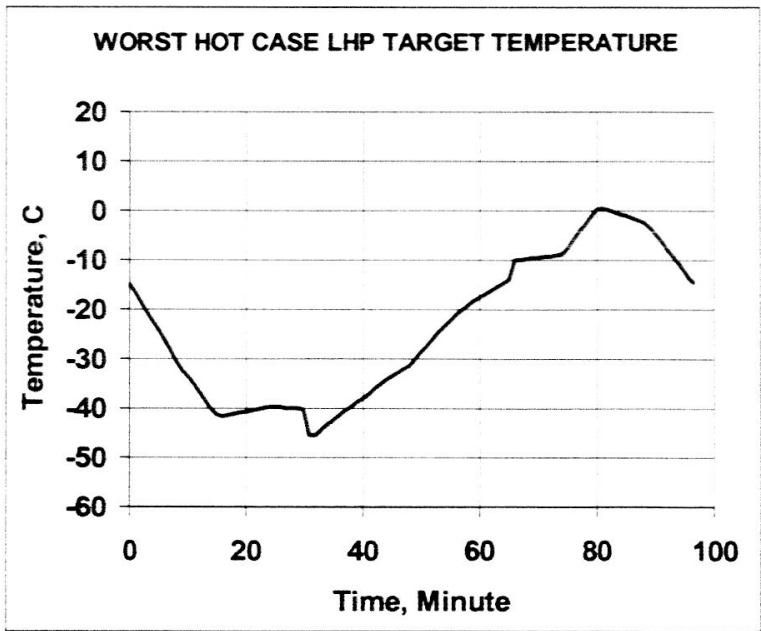

Figure 10. Worst Hot Case LHP CC Target Temperature.

\section{B. Worst Cold Case}

Figure 11 presents the worst cold case radiator target temperatures for the BAT radiator, comparing the aforementioned three values of heat transport by the LHP to the radiator. As in the worst hot case, the heat transport by the LHP to the radiator has a significant effect on the radiator target temperature. As the heat transport increases, the radiator target temperature decreases. The radiator target was cooled by liquid nitrogen. Therefore the coldest possible temperature for the target was only $-192^{\circ} \mathrm{C}$. This is why all the 3 curves bottom out at $-192^{\circ} \mathrm{C}$. When the 
target temperature is $-192^{\circ} \mathrm{C}$, the radiator temperature in the test is up to $5^{\circ} \mathrm{C}$ warmer than the equivalent radiator temperature for flight.

Figure 12 presents the worst cold case target tcmperatures for the LIIP CCs. The LHP CC heater controllers were set to $-15^{\circ} \mathrm{C}$ in the worst cold case.

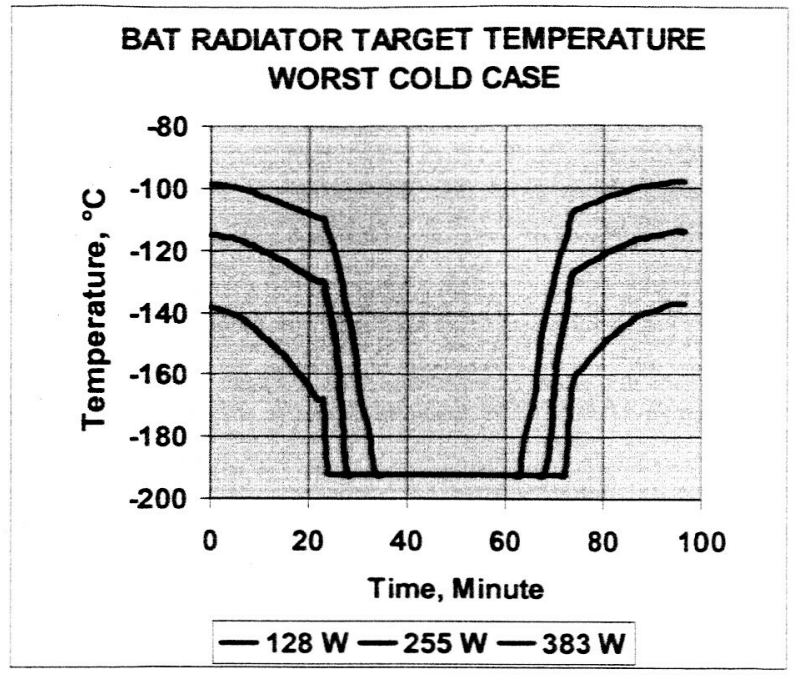

Figure 11. Worst Cold Case BAT Radiator Target Temperatures.

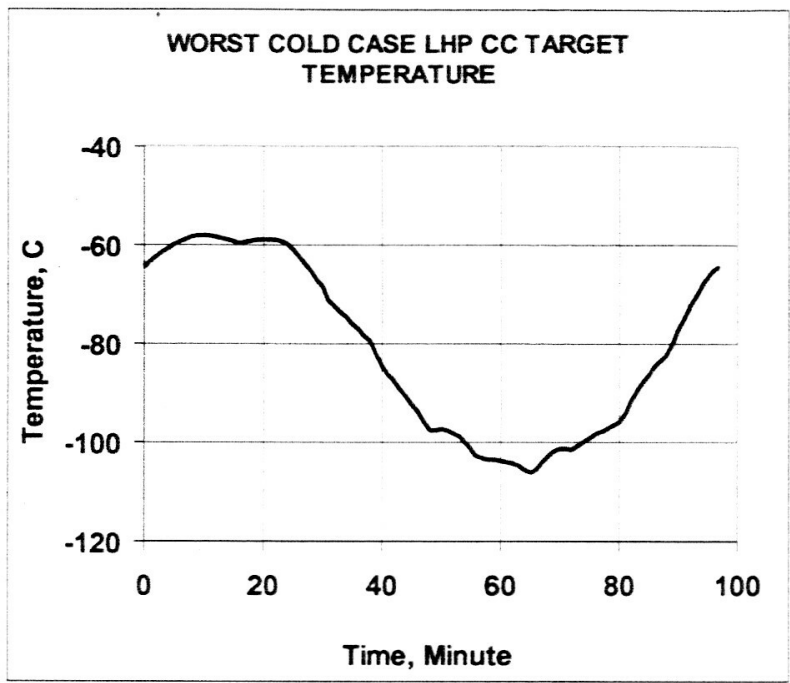

Figure 12. Worst Cold Case LHP CC Target Temperature.

\section{Cooldown and Warmup between Worst Hot Case in Sunlight and Worst Cold Case in Eclipse}

Figure 13 presents the radiator target temperatures for cooldown and warmup between worst hot case in sunlight and worst cold case in the eclipse, comparing the aforementioned three values of heat transport by the LHP to the radiator. As in the worst hot and worst cold cases the heat transport by the LHP to the radiator has a significant effect on the radiator target temperature. As the heat transport increases, the radiator target temperature decreases.

Figure 14 presents the LHP CC target temperatures for cooldown and warmup between worst hot case in sunlight and worst cold case in eclipse. The $\mathrm{CC}$ heater controller set point was set to $11^{\circ} \mathrm{C}$ in sunlight and $-15^{\circ} \mathrm{C}$ in the eclipse.

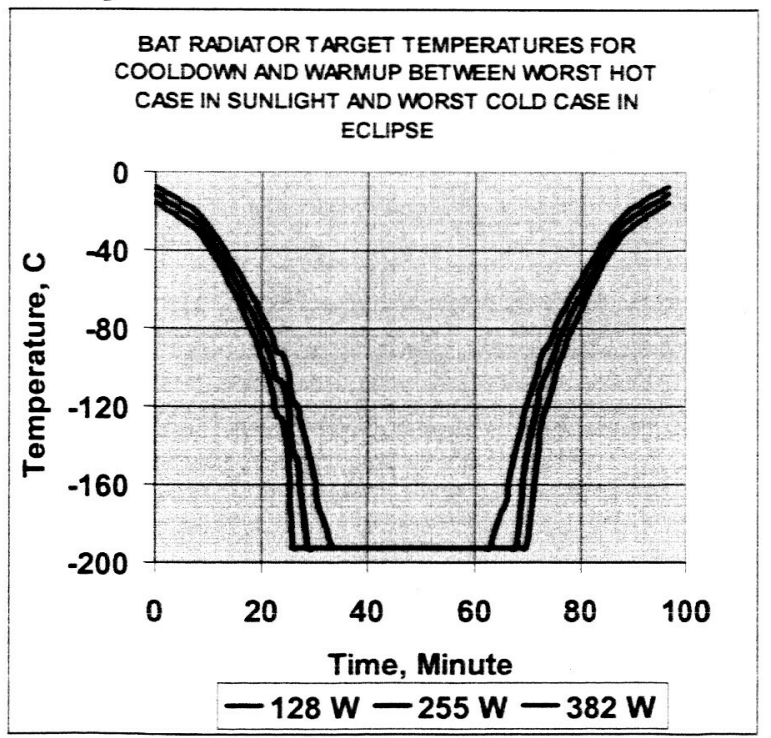

Figure 13. BAT Radiator Target Temperatures for Cooldown and Warmup between Worst Hot Case in Sunlight and Worst Cold Case in Eclipse.

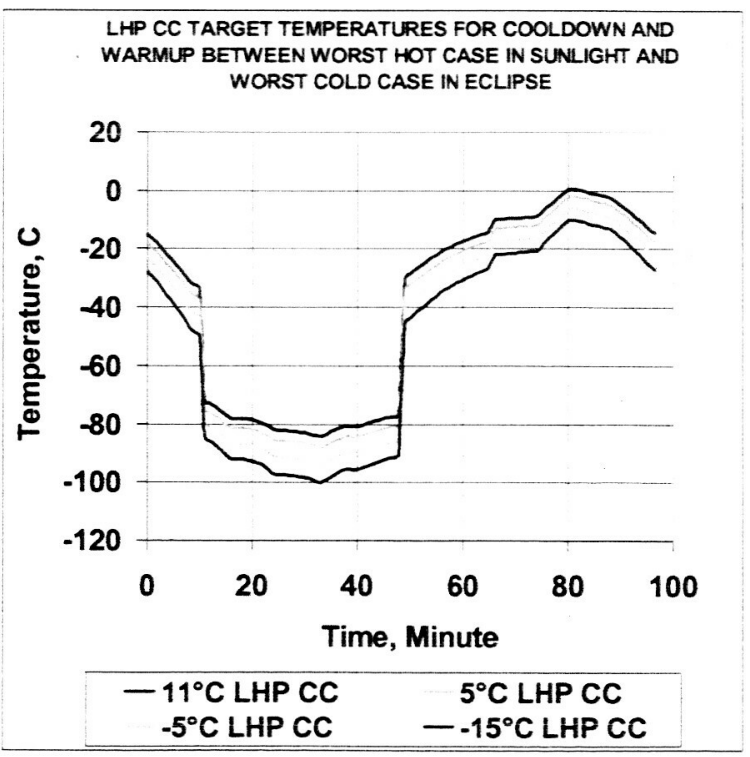

Figure 14. LHP CC Target Temperature for Cooldown and Warmup between Worst Hot Case in Sunlight and Worst Cold Case in Eclipse. 


\section{Effect of Target Emittance on Target and Radiator Temperatures}

As mentioned earlier, the black paint on the radiator target has a hemispherical emittance of 0.86 only, so that heat radiated from the radiator to the target is partially reflected back. From the equivalent sink temperatures in Figures 6 through 8, and the radiator target temperatures in Figures 9,11 and 13, the target temperature is as much as $30^{\circ} \mathrm{C}$ colder than the equivalent sink temperature.

\section{Conclusion}

In thermal performance verification testing of the BAT ETU LHP and flight LHPs in vacuum, the BAT radiator and LHP CCs radiated heat to sink targets. An analytical technique was developed to generate transient equivalent sink temperatures for the radiator and CCs. Also an analytical technique was developed to generate transient target temperatures for the radiator and CCs. In the technique of generating equivalent sink temperature, a GMM was run to generate environmental heat fluxes absorbed and radiation couplings first. Then a TMM was run to generate the equivalent sink temperatures. In the TMM, all the radiator and CC nodes are arithmetic nodes, all the radiator and $\mathrm{CC}$ nodes have no power dissipation, all the radiator and $\mathrm{CC}$ nodes have no conduction couplings, and there is no heat radiation through the radiator or $\mathrm{CC}$ thermal blankets. In the technique of generating sink target temperatures, the equivalent radiator temperature was first computed on a Microsoft EXCEL spreadsheet by using the transient equivalent sink temperature, heat transported by LHPs to the radiator, environmental heat fluxes absorbed, and total radiation coupling from the radiator to space and spacecraft components. The $\mathrm{CC}$ heater controller set point was also selected. Then the GMM was run to generate the radiation couplings between the radiator and its sink target, and that between the CCs and their targets. Using the transient equivalent radiator temperature, heat transported by LHPs to the radiator, and radiation coupling between the radiator and sink target, the transient target temperature for the radiator was computed on a Microsoft EXCEL spreadsheet. Using the CC heater controller set point, radiation couplings between the CCs and sink targets, and radiation couplings from the CCs to space and spacecraft components, the transient target temperature for the CCs was also computed on a Microsoft EXCEL spreadsheet.

Using these techniques, transient target temperatures for the radiator and CCs were generated for three thermal environmental cases: worst hot case, worst cold case, and cooldown and warmup between worst hot case in sunlight and worst cold case in the eclipse, and three different heat transport values: $128 \mathrm{~W}, 255 \mathrm{~W}$ and $382 \mathrm{~W}$. All these transient target temperatures were successfully implemented in the ETU LHP and flight LHP thermal performance verification tests in vacuum.

\section{References}

'Choi, M. K., "Thermal Design to Meet Stringent Temperature Gradient/Stability Requirements of Swift BAT Detectors," Paper No. AIAA-2000-2905, IECEC 2000, Las Vegas, NV, July 24-28, 2000.

${ }^{2}$ Choi, M. K., "Swift BAT Loop Heat Pipe Thermal System Characteristics and Ground/Flight Operation Procedure," Paper No. AIAA-2003-6077, $1^{\text {st }}$ International Energy Conversion Engineering Conference, Portsmouth, VA, Aug. 13-16, 2003. 